\title{
Case-finding for mental distress in primary health care: An evaluation of the performance of a five-item screening instrument ${ }^{*}$
}

\author{
Peter J. Chipimo, ${ }^{1,2 \#}$, Knut Fylkesnes ${ }^{2}$ \\ ${ }^{1}$ Department of Community Medicine, School of Medicine, University of Zambia, Lusaka, Zambia; \\ \#Corresponding Author: Peter.Chipimo@cih.uib.no \\ ${ }^{2}$ Centre for International Health, Faculty of Medicine and Dentistry, University of Bergen, Bergen, Norway; \\ Knut.Fylkesnes@cih.uib.no
}

Received 21 December 2012; revised 23 January 2013; accepted 7 February 2013

\begin{abstract}
Background: There is an increased interest in developing better and more accurate methods to recognize and manage mental health problems in primary care settings. Abbreviated screening instruments for mental distress are useful tools for research and clinical practice. The present study seeks to investigate whether only a few questionnaire items from the Self-ReportingQuestionnaire-10 (SRQ-10) can be a robust method in the screening for Mental Distress in Primary Health care. Methods: We compared the screening accuracy of a short, five-item (SRQ-5) version of the SRQ-10 with that of the SRQ-20, General Health Questionnaire 12 (GHQ-12) using the DSM-IV axis as a gold standard and analyzed its performance in different diagnostic entities. We also assessed the correlation, sensitivity and specificity between the 4 instruments. All the instruments were administered to 400 primary health care attendees. Results: The estimated prevalence of mental distress was $13.6 \%$ in the study sample (Depression $11 \%$, anxiety disorders $1.8 \%$ ). The SRQ-5 was highly correlated to SRQ-10 (0.923, $p<0.001)$, SRQ-20 (0.764, $p<0.001)$ and only moderately correlated to GHQ-12 (0.417, $p<0.001)$. The SRQ-5 had high properties for identifying mental distress. The AUC for overall mental distress was 0.925 while that for depression and anxiety were 0.915 and 0.849 respectively. Conclusion: This validation showed that in moving from SRQ with $\mathbf{1 0}$ or $\mathbf{2 0}$

\footnotetext{
*Competing interests: The authors declare that they have no competing interests.

Authors' contributions: PJC and KF both contributed to the analysis and drafting of the manuscript. PJC also contributed to the design and conduct of the study while KF contributed to the critical revision of manuscript and approval of final version.
}

items to one with merely 5 items, we do not seem to lose the screening prowess of the instrument. The SRQ-5 represents a simplified and less time-consuming screening instrument with strong performance characteristics. We therefore recommend it for inclusion into existing patient assessment protocols, thus enhancing case finding at primary health care level.

Keywords: Mental Distress; Screening Instruments; Validity; Primary Health Care; SRQ-5; SRQ-10; SRQ-20; GHQ12; DSM-IV; Zambia

\section{BACKGROUND}

Mental distress is a term used by both mental health practitioners and the users of mental health services. It is used to describe personal experiences of one's internal life that are commonly held to be out of the ordinary, troubling, confusing etc. [1]. It presents as a wide range of symptoms and has a wider scope than the related term mental illness. Some users of mental health services advocate for use of the term mental distress in describing their experience as they feel it better captures the sense of the uniqueness and personal nature of their experience, since everyone experiences distress at different times. The term also fits better with the social model of disability. Mental illness on the other hand, refers to a specific set of medically defined conditions. A person with mental distress may exhibit symptoms described as psychiatric, such as: depression and anxiety, without actually being 'ill' in a medical sense [1]. These symptoms may resolve without further medical intervention, although people who endure such symptoms in long run are more likely to be diagnosed with mental illness. Predictors known to induce mental distress include life situations such as: poor social and economic situation, bereavement, 
use of drugs or alcohol, abuse or accidents. This definition is not without controversy as some mental health practitioners would use the terms mental distress and mental illness interchangeably [1].

Mental health is an important foundation for attainment of intellectual, economic, emotional, social and educational well being [2]. Accordingly, mental ill health is a major contributor to the global burden of disease [3]. Estimates of the current prevalence of mental distress in Sub-Saharan Africa generally range between $6 \%$ and $20 \%$ of the adult population [4-6] though higher prevalence has also be reported in Ethiopia 49.1\% [7], South Africa $65.1 \%$ [8], and are even higher in some subgroups such as the elderly, the chronically ill and in institutionalized people $[9,10]$. The World Health Organization (WHO) states that a quarter of the world's population who have common forms of mental illness should be treated in Primary Health Care settings [3]. Despite the high prevalence of mental distress revealed in epidemiological studies, physicians in primary health care often overlook the salient symptoms of mental distress, especially in African countries [5,11,12]. Therefore a high number of patients who have mental distress do not receive any treatment. However, many screening instruments are available to aid in identification of the signs and symptoms of mental distress. These instruments have been developed for use in three major settings i.e. primary care, psychiatric outpatient services and for community surveys. It has been noted, however, that these instruments remain under utilized mostly due to the fact that most of them contain too many questionnaire items and have somewhat difficult likert system of scoring [13]. This study stems from efforts to improve the screening procedure in primary care. It also stems from the need to address the concerns of the general physicians, who are often pressed for time, for a convenient, accurate and brief instrument.

We have previously reported on the comparative validity of three screening instruments in detecting mental distress in a primary care setting using receiver-operatingcharacteristics as the quantitative measure of performance [13]. We also used the three instruments as concurrent criteria for each other and compared them against a gold standard, DSM-IV [13]. The three instruments studied were the SRQ-20, GHQ-12 and SRQ-10. The SRQ20 is a 20 item mental health questionnaire that was developed by the World Health Organization (WHO) [14]. The SRQ-10 is an abbreviated version of the SRQ-20 and is similarly based on a dichotomous response system. The GHQ-12 is a 12 item questionnaire designed for use in general practice of medicine and is also an abbreviated version on the original GHQ-60. These instruments have been validated in different setting and found to function well in the detection of mental distress [13-15]. Overall it was demonstrated that the SRQ-10 performed just as well as the SRQ-20 and was even better than the GHQ12 according to the ROC analysis in detecting mental distress.

In this paper, we extend our investigation of the SRQ by comparing the performance of its shortest version, the five item SRQ-5 with that of the SRQ-10, SRQ-20 and the GHQ using the DSM-IV as the gold standard. Receiver-Operating-Curves (ROC) is here used as the main technique for comparison.

\section{METHODS}

\subsection{Participants}

As has been previously reported elsewhere [13], a concurrent nested mixed methods design was used to assess 400 primary health care clinic attendees aged 16 years and above who were selected on random days from 4 different clinics in the city of Lusaka, Zambia between December 2008 and May 2009. The clinics were purposely selected within the city of Lusaka. Each clinic was sampled randomly on selected days, 3 times a week. On the selected days, interviews were conducted with consecutive clinic attendees at the clinic outpatients department. Participants were recruited based on their age, giving consent to participation as well as attendance to the out-patient department on the day of the study. Participants who were known to be psychiatric patients or those who were acutely or chronically ill or showed overt signs of psychiatric disorder were excluded from the study. The purpose of the study was explained to each participant by the research assistant and consent obtained. None of the participant approached declined being involved in the study. All participants were asked to answer a brief questionnaire concerning social and demographic characteristics as well as the SRQ-20 and GHQ 12 which were used as tools to identify probable "cases" of mental distress. Participants were then grouped as cases (based on scores of 7+ for SRQ-20 and 4+ for GHQ-12) or non-cases. They were then directed to one of the two general medical officers who held a clinical interview for the ailments that brought them to the clinic as well as conducting a psychiatric interview using the DSM-IV (gold standard). The medical officers used their clinical judgment along side the DSM-IV criteria to make the psychiatric diagnosis, and they were blinded as to the result of the initial screening with the SRQ-10 and GHQ-12. In depth interviews were also carried out with in a subsample of 28 participants nested within the quantitative sample. Based on the results of the screening, this sub-sample consisted of 14 cases and 14 non-cases of mental distress. These interviews were used to assess face and content validity of the screening tools [13]. 


\subsection{Screening Instruments and Diagnostic Interview}

The SRQ-20 was developed by the World Health Organisation (WHO) as a screening tool for common mental disorders primarily in primary health care settings in developing countries [14]. It consisted of 20 questions related to neurotic symptoms as opposed to psychotic symptoms in part due to the fact that for functional psychosis more active case finding in the community is required. Psychotic patients also tend to present to primary health care centres spontaneously and are often easily recognised by their pathognomonic features in most cases [14]. This instrument was not designed to specifically screen for depression or anxiety. The SRQ-20 has been validated in numerous settings with a widely accepted cut-off points of 7/8 [14].
The SRQ-10 is derived from the SRQ-20 which, in this study, was specially constituted with weighted sum of 10 symptom questions with a dichotomous response system (Yes/No) but neither probed for symptom severity nor designed to specifically screen for depression or anxiety. We have previously validated this tool [13] and used it in population based surveys in Zambia and yielded results that were comparable to other studies done elsewhere using the SRQ-20 [5,14]. It is a symptom inventory inquiring about the symptoms over the preceding 30 days presented as SRQ-10 items in Tables 1 and 2.

The SRQ-5 is an abbreviated version of the SRQ-10. The selection of the questionnaire items was based on the results from the validation study conducted in Zambia [13], and on consultations with a panel of psychiatrists

Table 1. Socio-demographic characteristics of study participants.

\begin{tabular}{|c|c|c|c|c|}
\hline & & \multicolumn{3}{|c|}{ Number (\%) of respondents } \\
\hline & & Male (N = 167) & Female $(N=233)$ & Total $(N=400)$ \\
\hline \multicolumn{5}{|c|}{ Characteristic } \\
\hline \multirow[t]{5}{*}{ Age } & $16-24$ & 31.7 & 36.6 & 34.6 \\
\hline & $25-29$ & 13.2 & 25.9 & 20.6 \\
\hline & $30-39$ & 29.9 & 25.9 & 27.6 \\
\hline & $40-49$ & 16.2 & 7.3 & 11.0 \\
\hline & $50+$ & 9.0 & 4.3 & 6.3 \\
\hline \multirow[t]{2}{*}{ Marital status } & Single & 44.3 & 30 & 36.0 \\
\hline & Married & 55.7 & 70 & 64 \\
\hline \multirow[t]{4}{*}{ Education } & Illiterate & 1.8 & 5.2 & 3.8 \\
\hline & Primary & 11.4 & 27.2 & 20.6 \\
\hline & Secondary & 60.5 & 53.0 & 56.1 \\
\hline & Tertiary & 26.3 & 14.7 & 19.5 \\
\hline \multirow[t]{3}{*}{ Wealth index } & low & 24.8 & 39.1 & 33.4 \\
\hline & middle & 33.3 & 33.5 & 33.4 \\
\hline & High & 41.8 & 27.4 & 33.2 \\
\hline \multirow[t]{4}{*}{ Language of interview } & English & 62.3 & 39.9 & 49.3 \\
\hline & Nyanja & 29.3 & 45.5 & 38.8 \\
\hline & Bemba & 6.0 & 10.3 & 8.5 \\
\hline & Other & 2.4 & 4.3 & 3.5 \\
\hline \multirow[t]{3}{*}{ Gold standard } & DSM-IV & 12.9 & 14.0 & 13.6 \\
\hline & Depression & 11.0 & 11.0 & 11.0 \\
\hline & Anxiety & 0.6 & 2.6 & 1.8 \\
\hline
\end{tabular}


Table 2. SRQ-10 items and the independently selected five and seven item combinations selected by six experienced medical practitioners.

\begin{tabular}{|c|c|c|}
\hline \multicolumn{2}{|c|}{ SRQ-10 Items: In the past 30 days... } & Item weight $^{\S}$ \\
\hline \multicolumn{2}{|l|}{ 1) Do you sleep badly? } & 1 \\
\hline \multicolumn{2}{|l|}{ 2) Do you cry more than usual? } & 1 \\
\hline \multicolumn{2}{|c|}{ 3) Do you find it difficult to enjoy your daily activities? } & 3 \\
\hline \multicolumn{2}{|c|}{ 4) Do you find it difficult to make decision? } & 1 \\
\hline \multicolumn{2}{|l|}{ 5) Is your daily life suffering? } & 3 \\
\hline \multicolumn{2}{|c|}{ 6) Are you unable to play a useful part in life? } & 3 \\
\hline \multicolumn{2}{|c|}{ 7) Has the thought of ending your life ever been on your mind? } & 5 \\
\hline \multicolumn{2}{|l|}{ 8) Do you feel tired all the time? } & 1 \\
\hline \multicolumn{2}{|l|}{ 9) Do you often have headaches? } & 1 \\
\hline \multicolumn{2}{|l|}{ 10) Is your digestion poor? } & 1 \\
\hline Selected SRQ-5 Items & 5-Item combination ${ }^{\#}$ & $\begin{array}{l}\text { 7-item combinations }{ }^{\#} \\
\text { (2 items in addition to the } 5 \text { item combination) }\end{array}$ \\
\hline Validation study ${ }^{*}$ & $1,3,5,6,9$ & - \\
\hline \multicolumn{3}{|l|}{ Medical practitioners } \\
\hline Psychiatrist I & $1,56,7,9$ & 7,8 \\
\hline Psychiatrist II & $1,3,5,89$ & 6,7 \\
\hline Psychiatrist III & $1,3,5,6,9$ & 7,8 \\
\hline General medical officer I & $1,3,6,8,9$ & 2,5 \\
\hline General medical officer II & $1,5,6,8,9$ & 4,7 \\
\hline General medical officer III & $1,2,6,7,9$ & 7,8 \\
\hline
\end{tabular}

*Selection based on: Chipimo PJ, Fylkesnes K. Comparative Validity of Screening Instruments for Mental Distress in Zambia. Clinical Practice \& Epidemiology in Mental Health. 2010;6:4-15. ${ }^{\S}$ Weights based on: Chipimo PJ, Fylkesnes K.Mental distress in the general population Zambia: Impact of HIV and social factors. BMC Public Health, 2009; 9:298. " Numbering based on: SRQ-10 Items.

and general practitioners [13]. The questions selected in this symptom inventory inquired about the following symptoms over the preceding 30 days: Do you sleep badly? Do you often have headaches? Do you find it difficult to enjoy daily activities? Are you able to play a useful part in life? Is your daily life suffering?

The General Health Questionnaire is a screening instrument originally designed for use in general practice but now found to be valid for detecting psychiatric morbidity in community surveys as well. [15] It contains 12 symptom questions scored on a four-point likert scale ranging (0 - $1-2-3)$ from much-less-then-usual to much-more-than-usual. However, in the analysis this scale is often collapsed to a dichotomous scale (0 - 0 - 1 1). Varied cut-off points have been used depending on the setting although cut-off point of $3+$ is widely accepted as indicative of psychiatric morbidity.

The Diagnostic and Statistical Manual of Mental Disorders (DSM) is the standard classification of mental disorders intended to be applicable for use across settings; inpatient-outpatient clinics, primary care, and in the community. In this study it was used as the gold standard. It has a diagnostic classification comprising a list of the mental disorders that best reflect the signs and symptoms that are afflicting the individual being evaluated. For each disorder, a set of diagnostic criteria indicating what symptoms must be present (and for how long) in order to qualify for a diagnosis are provided [16]. The use of these diagnostic criteria has been shown to increase diagnostic reliability (i.e., likelihood that different users will assign the same diagnosis [17]. The DSM-IV is widely accepted and used as the gold standard for psychiatric diagnosis in Zambia.

All the instruments were translated into Nyanja and Bemba as these are the most predominantly spoken languages and then subsequently back translated to English by bilingual translators from the linguistics department of the University of Zambia. This was to ensure validity in conceptual meaning. A team of three male and three female interviewers who had no experience in mental 
health care administered the SRQ-20 and the GHQ-12. They, however, all had previous experience administering questionnaires in other epidemiological studies. A three day training session was conducted in administering the instruments. This involved explanation and discussion of conceptual definitions of each item in the instruments and role playing. This was followed by a 1 day field test.

\subsection{Selection of SRQ-5 Items}

Firstly, based on the results obtained from the validation study of the SRQ-20 [13], the following 5-items were selected as being the best-subset of questions that were best understood by the participants: Do you sleep badly? Do you often have headaches? Do you find it difficult to enjoy daily activities? Are you able to play a useful part in life? Is your daily life suffering? This means that these questions were understood by the participants in a manner that was similar to the psychopathology the interviewers meant to unearth.

Secondly, we independently asked three psychiatrists with at least 3 years experience working in a psychiatric institution in Zambia and three general medical officers, with similar experience, working in primary health care setting to choose 5-items from the SRQ-10 which they regarded as being the most important indicators for mental distress. After this we further asked them to select another set of 2 questions from the remaining 5 items. These are presented in Tables $\mathbf{1}$ and 2. It was unanimously agreed among all the medical practitioners that Item 7 (suicidal ideation, for 1 month duration) on its own was a sign of major depression and that presence of this item alone even in the absence of the other items constitutes severe mental distress.

\subsection{Analytic Methods}

The validity of the SRQ-5 was examined using the specificity (rate of participants correctly identified as noncases) and sensitivity (rate of participants correctly identified as cases). Receiver-Operating-Curves (ROC) were computed using SPSS version 15 and the area under the curve (AUC) used to compare the performance of SRQ-5 with the other instruments (SRQ-10, GHQ-12) using DSM-IV as the gold standard. ROC was also used to compare the performance across of SRQ-5 across the different diagnostic groups (Depression and Anxiety). ROC permits the exploration of the entire range of sensitivities and specificities at each of the possible cut-off points through demonstrating sensitivity at the y-axis and 1specificity at the $\mathrm{x}$-axis. Reliability was assessed using Cronbach's alpha (Internal consistency). Further independent t-tests were performed to compare the instruments by sex and age and a Pearson's Chi-square was computed to compare the psychiatric diagnosis in the same groups.

\section{RESULTS}

In all 400 participants, visiting the primary care (PHC) centers for various medical reasons, completed the SRQ-20 and the GHQ-12 and also completed the clinical interview (using the DSM-IV) administered by the medical officer. Ten respondents were not included because they refused the clinical interview, however, and no significant differences appeared between the total sample and the participants that refused the clinical interview in sex ratio, wealth status, marital status and educational attainment. Almost half of the respondents preferred English as the language for the interview, while the others preferred Nyanja (38.8\%) and Bemba (8.5\%). The interviewed sample tended to be women (58.3\%), younger than 40 years $(82.7 \%)$ and married (70\%). The male participants were on average 32 years $(\mathrm{SD}=11.1)$. About $56 \%$ of them were married and $61 \%$ of them had secondary school education. Female participants averaged 29 years $(S D=9.4)$ and had an average of more than 8 years of education (secondary $56 \%$ vs. tertiary 19.5\%) while $3.8 \%$ were illiterate. The estimated prevalence of mental distress was $13.6 \%$ (Depression 11\%, anxiety disorders 1.8\%). Table 1 shows the summary of the sociodemographic characteristics

Table 3 shows the correlation matrix of the screening instruments. The results show that SRQ-5 is highly correlated to SRQ-10 (0.923, p < 0.001), SRQ-20 (0.764, p $<0.001)$ and only moderately correlated to GHQ-12 (0.417, $\mathrm{p}<0.001)$. Slightly better correlation was seen between GHQ-12 and SRQ-10 (0.515, p < 0.001) and SRQ-20 (0.593, p < 0.001). All correlations were significant at $\mathrm{p}<0.001$. In Table 4 , areas under the receiver operating characteristic curve (AUC) was calculated for each of the 4 screening instruments and compared for the different diagnostic groups. These diagnostic groups inclued: 1) depression and 2) anxiety disorders. By this measure SRQ-5 is almost equal to SRQ-10 and SRQ-20. However it out-performs GHQ-12 for detection of overall mental distress and depression and equals it in the detection of anxiety. All these AUC are significant at $\mathrm{p}<$

Table 3. Correlation matrix for SRQ-5, SRQ-10, SRQ-20 and GHQ-12.

\begin{tabular}{ccccc}
\hline Screening instrument & 1 & 2 & 3 & 4 \\
\hline SRQ-5 & 1 & & & \\
SRQ-10 & 0.923 & 1 & & \\
SRQ-20 & 0.764 & 0.846 & 1 & \\
GHQ-12 & 0.417 & 0.515 & 0.593 & 1 \\
\hline
\end{tabular}


Table 4. Performance of the questionnaires: Areas under the ROC curve.

\begin{tabular}{ccccc}
\hline \multirow{2}{*}{ Criterion diagnosis } & SRQ-5 & SRQ-10 & SRQ-20 & GHQ-12 \\
\cline { 2 - 5 } & AUC (S.E) & AUC (S.E) & AUC (S.E) & AUC (S.E) \\
\hline Overall Mental Distress & $0.925(0.021)$ & $0.959(0.015)$ & $0.951(0.014)$ & $0.811(0.037)$ \\
Male & $0.964(0.020)$ & $0.994(0.005)$ & $0.989(0.007)$ & $0.807(0.059)$ \\
15 - 29 years & $0.896(0.032)$ & $0.931(0.025)$ & $0.921(0.024)$ & $0.813(0.047)$ \\
30 - 67 years & $0.886(0.038)$ & $0.961(0.021)$ & $0.967(0.013)$ & $0.835(0.052)$ \\
Depression & $0.966(0.017)$ & $0.963(0.020)$ & $0.945(0.023)$ & $0.795(0.052)$ \\
Anxiety & $0.915(0.018)$ & $0.951(0.018)$ & $0.931(0.018)$ & $0.774(0.043)$ \\
\hline
\end{tabular}

*All figures $\mathrm{p}<0.001$.

0.001. Figures 1-3 shows a graphic representation of the performance of the screening instruments, areas under the ROC curve. From the figures it is clear that the SRQ perform very well with curves that are similar in pattern across the criterion diagnosis. Furthermore, no major differences are noted when compared by sex and age.

Table 5 shows the AUC values when ROC is applied to the individual items that make up the SRQ-5. Each of the 5 items contributes some diagnostic information across all the criterion diagnosis. The table shows that each question performs well in the diagnosis of depresssion. The best contributor being the question: Do you sleep badly? (0.836, SE = 0.037) and the least being; Do you often have headaches? (0.606, SE $=0.045)$. All AUC values for detection of overall mental distress and depression for each of the SRQ-5 items were significant at $\mathrm{p}<0.05$ when compared to the full scale of the SRQ-10. What is explored here is simply the range of the response frame itself for each of the five items. Addition of more items to the 5 did not seem to improve the performance of the SRQ-5.

Table 6 shows the results of the analysis which focused on the ability of the SRQ-5 to detect mental distress at different cut-off points. The sensitivity, specificity, positive predictive values, and negative predictive and kappa's values of the scales with different cut-off points are here shown. The most appropriate cut-off point was a trade off between sensitivity and specificity. Since it is meant to be used as screening instruments, the optimal cut-off point is one with high sensitivity and an acceptable specificity. The optimal cut-off for SRQ-5 was $4 / 12$ with sensitivity 0.87 and specificity 0.85 . Further analysis by sex and gender or by criterion diagnosis did not reveal any significant differences in cut-off points. Practically speaking this means a patient presenting with either, poor sleep, daily life suffering or inability to play a useful part in life and any one of the other symptoms would be classified as being mentally distressed. A patient presenting with only suicidal ideation as a lone symptom or in combination with other symptoms would also be classified as probable case.

\section{DISCUSSION}

This is the first study examining the validity of the SRQ-5 in primary care and providing the DSM-IV diagnoses as a gold standard. The SRQ-5 had an AUC 0.925 and did not differ significantly from the SRQ-10 and the SRQ-20 but performed slightly better than the GHQ-12 (AUC; 0.925 vs. 0.811 respectively). Comparing the patterns of the ROC curves showed no significant difference in its performance by gender or age. The results show that SRQ-5 performed better for detecting diagnosis of depression than for anxiety and are in agreement with the content of the items in the questionnaire (AUC 0.915 depression vs. 0.849 anxiety). Analysis of item by item performance revealed that the items: do you sleep badly, and do you find it difficult to enjoy daily activities? Performed best across all diagnostic criteria. With respect to a cut-off point, ROC does not seem to suggest different cut-off points based on the diagnostic criterion. Using the weighted sum of scores for the items suggests that the best balance between sensitivity and specificity is a cutoff point of 4/12. Based on this cut-off point the sensitivity is 0.87 and the specificity is 0.85 . For general practitioners, this would mean that a patient presenting with either, poor sleep, daily life suffering or inability to play a useful part in life and any one of the other symptoms would be classified as being mentally distressed. Further a patient presenting with only suicidal ideation as a lone symptom or in combination with other symptoms would also be classified as probable case.

We compared the screening capability of the SRQ-5 with that of 3 validated tools. The SRQ-20 and GHQ-12 are widely validated tools and have been found to be acceptable in different settings and population groups [14, 15]. We have previously shown that the SRQ-10 is an acceptable screening tool with comparable, if not better, 


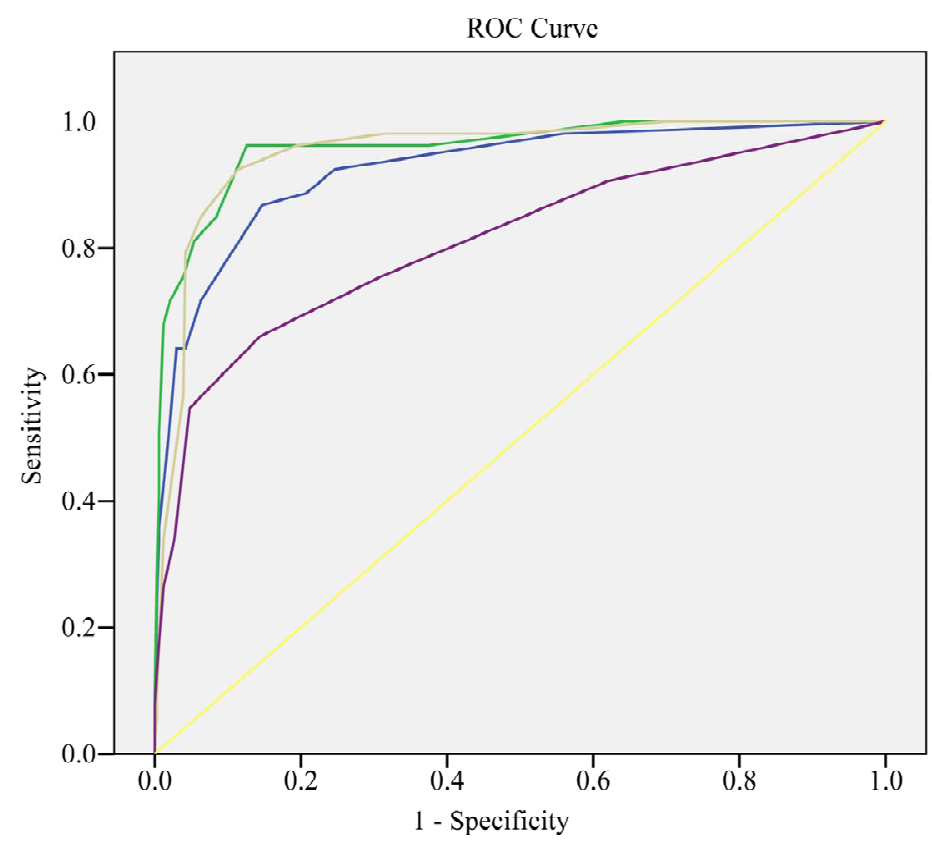

Source of the Curve

SRQ5 fullscale

SRQ10SUM (0-20)

SRQ20SUM $(0,1)$

- GHQ full scale

Reference Line

Figure 1. ROC for detection of overall Mental Distress for SRQ-5, SRQ-10, SRQ-20 and GHQ-12.

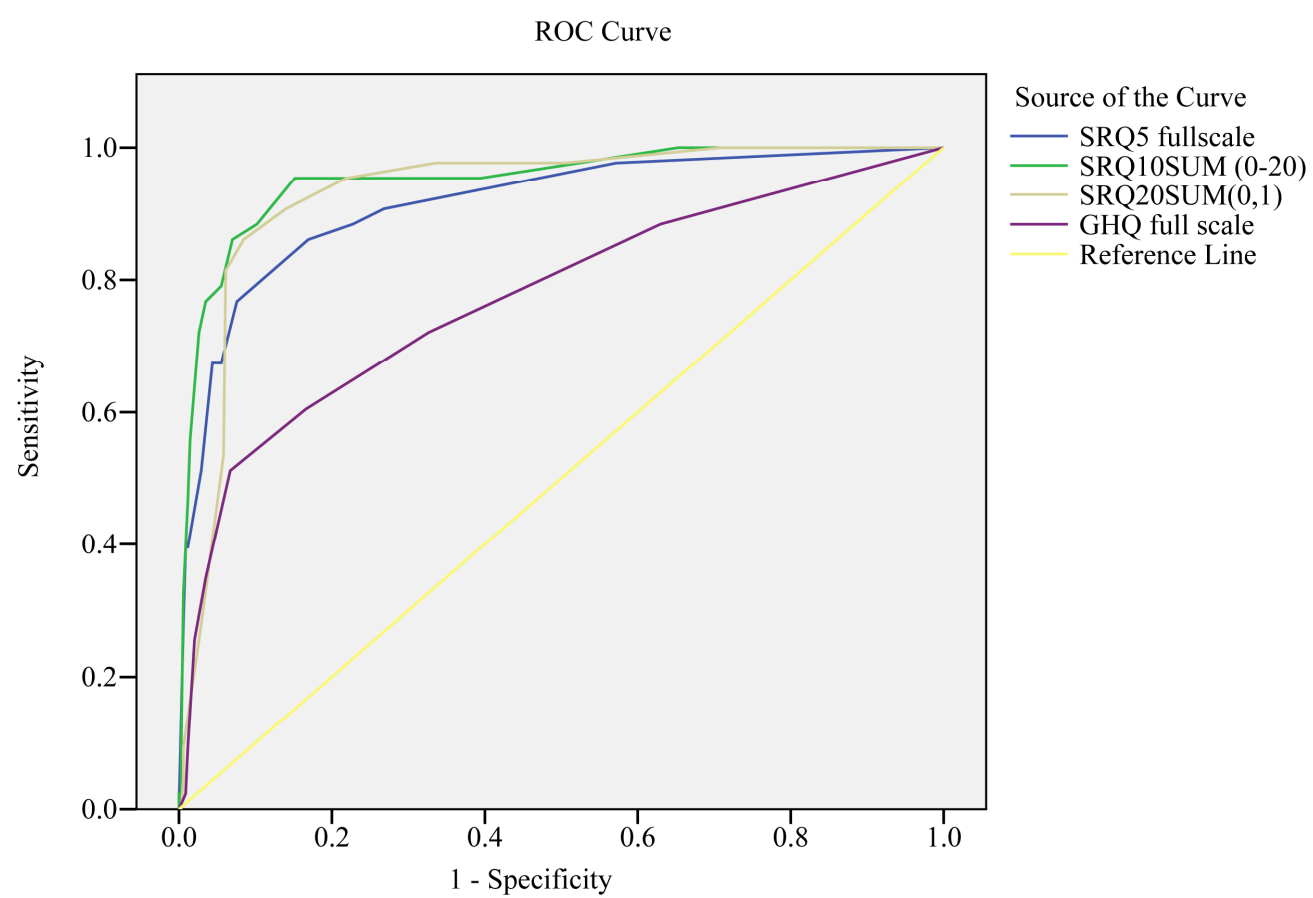

Figure 2. ROC for detection of depression SRQ-5, SRQ-10, SRQ-20 and GHQ-12.

screening characteristics than the SRQ-20 and the GHQ12 [13]. A number of other studies have been conducted world-over which have argued for the use of shorter screening tools for mental distress. Although we could not find directly comparable studies that have used the SRQ-5, we feel that the results are comparable to other studies since the content base of these other tools are similar. In their study aiming at validating the abbrevi- ated MHI-5 version, Rumpf et al. [18] demonstrated acceptable AUC for the MHI-5 using DSM-IV as the gold standard. They concluded by recommending the MHI-5 as a screening tool for mood disorders. Similarly, Jacobsen et al. [19] reported a strong association between the abbreviated GHQ-4 version and the full GHQ-20 and that this was similar by all examined subgroups of the study population. They thus concluded that very short 


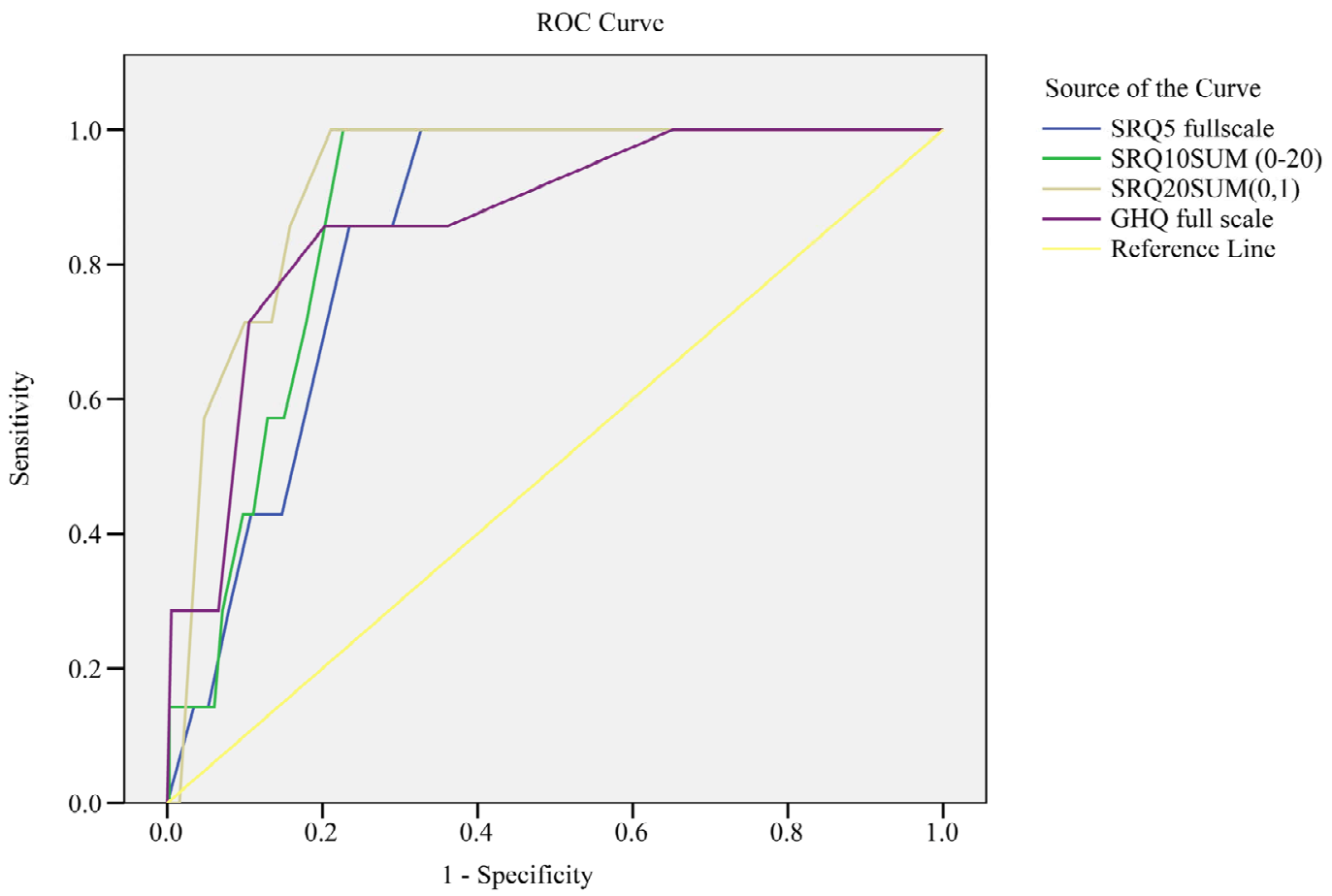

Figure 3. ROC for detection of anxiety SRQ-5, SRQ-10, SRQ-20 and GHQ-12.

Table 5. Performance of the single SRQ-5 Items: Areas under the ROC curve.

\begin{tabular}{ccccccc}
\hline Criterion diagnosis & SRQ-10 full scale & \multicolumn{5}{c}{ SRQ-5 Individual items } \\
\hline & AUC (SE) & Sleep AUC (SE) & Enjoy AUC (SE) & Suffer AUC (SE) & Useful AUC (SE) & Headache \\
AUC (SE) \\
Overall Mental Distress & $0.959(0.015)$ & $0.815(0.037)^{\mathrm{c}}$ & $0.826(0.035)^{\mathrm{c}}$ & $0.779(0.041)^{\mathrm{c}}$ & $0.709(0.045)^{\mathrm{c}}$ & $0.623(0.040)^{\mathrm{a}}$ \\
Depression & $0.951(0.018)$ & $0.836(0.037)^{\mathrm{c}}$ & $0.813(0.039)^{\mathrm{c}}$ & $0.790(0.044)^{\mathrm{c}}$ & $0.726(0.049)^{\mathrm{c}}$ & $0.606(0.045)^{\mathrm{a}}$ \\
Anxiety & $0.879(0.030)$ & $0.693(0.080)$ & $0.757(0.100)$ & $0.637(0.118)$ & $0.589(0.120)$ & $0.689(0.086)$ \\
\hline
\end{tabular}

${ }^{\mathrm{a}} \mathrm{SRQ}-10$ vs SRQ-5 item $\mathrm{p}<0.05$; ' $\mathrm{b}$ SR-10 vs SRQ-5 item $\mathrm{p}<0.01$; 'SRQ-10 vs SRQ-5 item $\mathrm{p}<0.001$.

Table 6. Sensitivity and specificity of SRQ-5 for overall Mental Distress.

\begin{tabular}{|c|c|c|c|c|c|c|c|c|}
\hline $\begin{array}{l}\text { Screening } \\
\text { Instrument }\end{array}$ & Cut-off & Sensitivity & Specificity & PPV & NPV & $\begin{array}{c}\% \text { of cases } \\
\text { screened correctly }\end{array}$ & k & $\%$ cases \\
\hline \multirow[t]{5}{*}{ SRQ-5 } & 4 & 0.87 & 0.85 & 0.48 & 0.98 & 85.4 & 0.54 & 24.3 \\
\hline & 5 & 0.72 & 0.94 & 0.64 & 0.96 & 90.8 & 0.63 & 15.0 \\
\hline & 6 & 0.64 & 0.96 & 0.70 & 0.97 & 91.6 & 0.63 & 12.0 \\
\hline & 7 & 0.64 & 0.97 & 0.77 & 0.95 & 92.6 & 0.65 & 11.0 \\
\hline & 8 & 0.49 & 0.98 & 0.81 & 0.92 & 91.6 & 0.54 & 8.0 \\
\hline
\end{tabular}

versions of the GHQ questionnaires could be used effectively in population surveys on mental distress [19]. Friedman et al. [20] examined the criterion and construct validity of the five-item (MHI-5) of the SF-36. Using AUC they demonstrated that the MHI-5 had adequate criterion validity and satisfactory sensitivity and specificity. They concluded that MHI-5 exhibited ade- quate properties for measuring presence of depression [20].

Though the cited studies were not particularly validating the SRQ, the item bases are very similar and focus more on neurotic disorders. It would suffice to say from the reviewed literature that even a shorter, and only slightly less powerful screening tool, maybe used in 
place of a longer more comprehensive questionnaire to achieve acceptable results [21].

The authors selected SRQ over the other "competing tools” (GHQ, CIS-R, PHQ-9, CHAT) because it was derived from a wide variety of cultural backgrounds and was endorsed in 1994 by WHO for use in primary health care in developing countries. The instrument also assumes that the population of interest may have poor infrastructure, have low literacy levels which make phone interviews and self completion infeasible, as is often the case in developing countries. The use of an abbreviated version of the SRQ therefore adds dimension and depth to other studies which have used the SRQ in broader social science research.

The selection criteria of the 5 items of the SRQ- 5 took on a two fold approach. The first being based on the validation study of the SRQ conducted in Zambia [13], which rendered half of the questions in the SRQ-20 invalid based on misconceptualisation of questions on the part of the participants. The other questions, though found to capture the psychopathology sort by the medical practitioner, were also found to be invalid in the face of intercurrent illness or co-morbidity. We therefore did not include these questions in the SRQ-5 [13]. The second strategy involved eliciting expert advice from 3 experienced psychiatrists and 3 general medical practitioners. The items selected by the medical practitioners were similar in at least 4 of the 5 questions. They additionally unanimously agreed that suicidal ideation of at least 1 month constituted severe depression and so even when found as alone symptoms would be enough to make a diagnosis of mental distress. The fact that these two selection criteria yielded somewhat similar results, further added impetus to the robustness of this selection strategy. Similar selection strategy was used by Jacobsen et al. and yielded comparable results [19].

Scale developers have traditionally evaluated performance of screening tools by comparing the sensitivity and specificity by gender and age. In our study the validity coefficients did not seem to differ in these sub-groups. This would suggest that it is unnecessary to use different cut-off points in these groups. These results are somewhat similar to other studies investigating abbreviated screening tools. Cleary et al. [22], in their study to evaluate the use of mental health screening scales in primary care also found no statistical difference by gender or sex. They however cautioned that the interpretation of these results is not clear cut as literature on this subject is not in agreement. They advised that the presence of these gender and sex differences [23] should be born in mind as test results could differ substantially by patient group, even when the overall validation is similar [23].

In conclusion, this validation showed that in moving from SRQ with 10 - 20 items to one with merely 5 items, we do not seem to lose the screening prowess of the instrument. The SRQ-5 represents a simplification of the mental health screening process while exhibiting strong performance characteristics. This has implications in the clinical settings as well as in the policy formulation sector. It is of importance in the clinical setting in that such an abbreviated scale allows for its administration without being overly time consuming. In as much as the SRQ-5 is unable to provide an exact diagnostic classification, it has been shown to be a reliable flag for detecting the common symptom pathways (mental distress) that then manifest as specific psychiatric diagnosis, especially in primary health care. This provides an entry point to specialized psychiatric treatment for those who are diagnosed with mental illness, or indeed an entry point to counseling services for those with milder forms of mental distress. It is as such a step forward in ensuring that as many people get the mental health care they need. Additional impetus for such an instrument is added by the growing body of evidence for the consistent linkage between mental distress and other chronic and acute illness and the recognition that feasible actions within a primary health setting which identifies and treats mental distress have a long-run better outcome for other intercurrent illness. It is important from a policy perspective because, when used in population surveys, it allows for access to prevalence data that is critical to formulation of cogent national mental health policies as well as to the success of such policies. Further the need for a cost-effective instrument to measure mental health, especially in developing countries, has increased over the last decade. The paper provides a method of measuring mental distress that is cost-effective (in-terms of time and level of human resource) and provides a window which allows for access to assurance of specific annual budget al location to mental health.

\section{ACKNOWLEDGEMENTS}

The authors would like to acknowledge Dr Francis Simenda (Director, Chainama Hospital) for his expert help with the screening tools and valuable advise. We would also like to acknowledge the financial support from Norwegian Programme for Development, Research and Education (NUFU).

\section{REFERENCES}

[1] Goldberg, D. (2000) Distinguishing mental illness in primary care. Mental illness or mental distress? British Medical Journal, 321, 7273. doi:10.1136/bmj.321.7273.1412

[2] Williams, D.R., Herman, A., Stein, D.J., Heeringa, S.G., Jackson, P.B., Moomal, H., et al. (2008) Twelve-month mental disorders in South Africa: Prevalence, service use and demographic correlates in the population-based South African Stress and Health survey. Pschological Medicine, 


\section{8, 211-220. doi:10.1017/S0033291707001420}

[3] WHO (2001) World health report-mental health: New understanding, new hope. WHO, Geneva.

[4] Giel, R., Gezahegn, Y. and Vann-Luuk, J.N. (1968) Psychiatric morbidity in 200 Ethiopian medical outpatients. Psychiatr Neural Neurochir, 71, 169-176.

[5] Chipimo, P.J. and Fylkesnes, K. (2009) Mental distress in the general population: Impact of HIV and social factors. BMC Public Health, 9, 298.

[6] Kabede, D., Alem, A. and Rashid, E. (1999) The prevalence and socio-demographic correlates of mental distress in Addis Ababa, Ethiopia. Acta Psychiatr Scand, 100, 510. doi:10.1111/j.1600-0447.1999.tb10687.x

[7] Tesfaye, A. (2009) Prevalence and correlates of mental distress among regular undergraduate students of Hawassa University: A cross sectional survey. East African Journal of Public Health, 6, 1. doi:10.4314/eajph.v6i1.45755

[8] Abbo, C., Ekblad, S., Waako, P., Okello, E. and Muhwezi, W.S.M. (2008) Psychological distress and associated factors among attendees of traditional healing practices in Jinja and Iganga districts, Eastern Uganda: A cross-sectional study. International journal of Mental Health Systems, 2, 1-9.

[9] Ngoma, M.C. and Prince, M.A.M. (2003) Common mental disorders among those attending primary health clinics and traditional healers in urban Tanzania. The British Journal of Psychiatry, 183, 349-355. doi:10.1192/bjp.183.4.349

[10] Ciesla, J.A. and Robert, J.E. (2001) Meta-analysis of the relationship between HIV infection and the risk for depressive disorders. American Journal of Psychiatry, 158, 725-730. doi:10.1176/appi.ajp.158.5.725

[11] Jacob, K.S. (2001) Community care for people with mental disorders in developing countries: Problems and possible solutions. The British Journal of Psychiatry, 178, 296-298. doi:10.1192/bjp.178.4.296

[12] Kigozi, F., Ssebunnya, J., Kizza, D., Ndyanabangi, S., Green, A., Omar, M., et al. (2008) A situation analysis of the mental health health system in Uganda: Mental health \& poverty project.

[13] Chipimo, P.J. and Fylkesnes, K. (2010) Comparative va- lidity of screening instruments for mental distress in Zambia. Clinical Practice \& Epidemiology in Mental Health, 6, 4-15. doi:10.2174/1745017901006010004

[14] WHO (1994) A user's guide to the Self-Reporting Questionnaire. World Health Organisation, Geneva.

[15] Goldberg, D.P., Gater, R., Sartorious, N., et al. (1997) The validity of two versions of GHQ in the WHO study of mental illness in general health care. Psychological Medicine, 27, 191-197. doi:10.1017/S0033291796004242

[16] American Psychiatric Association (2000) Diagnostic and statistical manual of mental disorders (DSM-IV). 4th Edition, American Psychiatric Association, Washington DC.

[17] Mario, M., Wolfgang, G., Juan-Jose, L. and Norman, S. (2002) Psychiatric diagnosis and classification. 3rd Edition, In: Sons JW, editor..

[18] Rumpf, H., Meyer, C., Hapke, U. and Ulrich, J. (2001) Screening for mental health: Validity of the MHI-5 using DSM-IV Axis I psychiatric disorders as gold standard. Psychiatry Research, 105, 243-253. doi:10.1016/S0165-1781(01)00329-8

[19] Jacobsen, B.K., Hasvold, G., Hansen, H. and Hansen, V. (1995) The general health questionnaire: How many items are really necessary in population surveys? Psychological Medicine, 25, 957-961. doi:10.1017/S0033291700037442

[20] Friedman, B., Heisel, M. and Delavan, R. (2005) Validity of the SF-36 five-item mental health index for major depression in functionally impaired, community-dwelling elderly patients. Journal of American Geriatrics Society, 53, 1978-1985. doi:10.1111/j.1532-5415.2005.00469.x

[21] Berwick, D.M., Murphy, J.M., Goldman, A., Ware, J.E., Barsky, A.J. and Weinsten, M.C. (1991) Performance of a five-item mental health screening test. Medical Care, 29, 169-176. doi:10.1097/00005650-199102000-00008

[22] Cleary, P.D., Bush, B.T. and Kessler, L.G. (1987) Evaluating the use of mental health screening scales in primary care setting using receiver operating characteristics curves. Medical Care, 25, S90-S98. doi:10.1097/00005650-198712001-00009

[23] Mari, J. and Williams, P.A. (1986) Validity study of a psychiatric screening questionnaire (SRQ-20) in primary care in the city of Sao Paulo. British Journal of Psychiatry, 148, 23-26.doi:10.1192/bjp.148.1.23

\section{LIST OF ABBREVIATIONS}

SRQ-20 = Self-Reporting Questionnaire 20;

SRQ-10 = Self-Reporting Questionnaire-10;

SRQ-5 = Self-Reporting Questionnaire-5;

SRQ's = Self-Reporting Questionnires;

GHQ-12 = General Health Questionnaire;

MHI = Mental Health Interview;

DSM-1V = Diagnostic and Statistical Manual of

Mental Disorder 4th Edition;

ROC = Receiver Operating Characteristics;

AUC = Area under the Curve;

$\mathrm{SE}=$ Standard Error;

NPV = Negative Predictive Value;

$\mathrm{PPV}=$ Positive Predictive Value 